\title{
Photosynthetic response and transcriptomic profiling provide insights into the alkali tolerance of clone halophyte Leymus chinensis
}

\author{
H. WANG ${ }^{*}$, Y. XIANG ${ }^{*}$ L.H. LI**, N. BHANBHRO**, C.W. YANG ${ }^{* *}$, and Z. ZHANG ${ }^{*}+$ \\ Department of Agronomy, Jilin Agricultural University, 130118 Changchun, China* \\ Key Laboratory of Molecular Epigenetics of Ministry of Education, Northeast Normal University, \\ 130024 Changchun, China**
}

\begin{abstract}
Alkali stress is one of the important factors in restricting agriculture production. Leymus chinensis is constructive halophyte species in alkalized grassland in China. In order to investigate the gene expression response of L. chinensis to alkali stress, we used PacBio technology to obtain reference full-length transcript sequences for transcriptomic analysis of alkali stress response. In order to elucidate the alkali tolerance mechanisms of L. chinensis, we measured the photosynthetic parameters, concentrations of ions and compatible solutes, chloroplast ultrastructure and anatomy of control and stressed plants. Our results showed that L. chinensis shares many alkali-tolerance mechanisms with glycophytes. Higher stability of photosynthetic apparatus under alkali stress may be prominent alkali-tolerance trait of L. chinensis. L. chinensis may have a strong capacity to decline the toxicity of $\mathrm{Na}^{+}$to organelles and cytoplasmic proteins. Enhanced expression of dehydrin and $L E A$ genes and increased accumulation of carbohydrates may contribute to the development of $\mathrm{Na}^{+}$-specific stress tolerance of L. chinensis under alkali stress.
\end{abstract}

Additional key words: osmotic adjustment; PacBio sequencing; pigments; RNAseq.

\section{Introduction}

Soil salinization and alkalization are important environmental factors restricting agriculture production in the world. In saline soil, harmful salts mainly consist of $\mathrm{NaCl}$, $\mathrm{Na}_{2} \mathrm{SO}_{4}, \mathrm{NaHCO}_{3}$, and $\mathrm{Na}_{2} \mathrm{CO}_{3}$. About $46 \%$ of saline soils contain only the neutral salts $\mathrm{NaCl}$ and $\mathrm{Na}_{2} \mathrm{SO}_{4}$, but the remaining 54\% contain both the neutral and alkaline salts (sodic soil) (Tanji 1990). Our previous studies have demonstrated that alkaline salt stress had strong destructive effects to plants compared to the neutral salt stress of the same salinity (Yang et al. 2007, 2008b). Alkalization is much more destructive to plants and soil than salinization. For example, in northeast China, about $70 \%$ of grasslands was alkalized (Tanji 1990). These alkaline soils contain high concentrations of $\mathrm{NaHCO}_{3}$ and $\mathrm{Na}_{2} \mathrm{CO}_{3}$, which can not only cause soil compaction and mineral element precipitation but also can destroy the chemical structure of root cell membrane. Only few alkali-tolerant halophytes can live in heavily alkalized grasslands. However, this serious environmental problem has been ignored and always is confused with 'salt-alkaline stress'. In order to reveal the adaptive mechanisms of plant to natural alkalized soils, we must distinguish between salt stress and alkali stress and pay more attention to plant alkali tolerance.

In the past 30 years, salt stress was one of the research hotspots in plant stress biology. A great progress was achieved in ion transport, signal transduction, hormone regulation, and other fields (Flowers and Yeo 1995, Munns and Tester 2008, Flowers et al. 2010, Kaashyap et al. 2017, Rozentsvet et al. 2017, Zhang et al. 2018, Zeng et al. 2018, Wang et al. 2019, Zhu et al. 2019). Although soil alkalization has caused serious ecological and agricultural problems in some area of northeast China (Tanji 1990), few studies focus on alkali stress. These alkali stress researches had focused on transgenesis (Wang et al. 2016, He et al. 2017), organic acid metabolism (Ma et al. 2017), physiology and gene expression (Tanji 1990, Zhang et al. 2013, Jia et al. 2019), metabolome (Guo et al. 2016), proteome (Yu et al. 2013, Zhang et al. 2016, Zhao et al. 2019), and microarray analysis (Wang et al. 2007a). Most of these descriptive works focused on glycophytes, and only few studies reported alkali tolerance of halophytes (Yang et al. 2007, 2008a; Li et al. 2010, Yin et al. 2019). To date, almost all molecular mechanisms

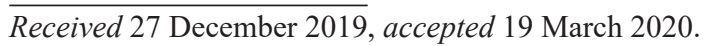

+Corresponding author; e-mail: zhangzhian6412@163.com

Abbreviations: DEG - differentially expressed gene; DM - dry mass; $E$ - transpiration rate; FM - fresh mass; GDH - glutamate dehydrogenase; GS - glutamine synthetase; $g_{\mathrm{s}}$ - stomatal conductance; HKT - high-affinity potassium transporter; KEGG - Kyoto Encyclopedia of Genes and Genomes; LEA - late embryogenesis abundant; NCL - sodium/calcium exchanger; NHX - sodium/ hydrogen exchanger; NPF - NRT1/PTR FAMILY; NRT - high-affinity nitrate transporter; $P_{\mathrm{N}}$ - net photosynthetic rate.

Acknowledgements: This work was supported by the National Natural Science Foundation of China $(31802114,31971762)$, and the China Postdoctoral Science Foundation (2017M610197, 2018T110262). 
of salt tolerance and alkali tolerance were discovered in rice and Arabidopsis (Wu et al. 2018, Flowers et al. 2019, Ganie et al. 2019). Most botanists believe that halophytes and glycophytes employ different mechanisms to resist salt stress and alkali stress. In addition, halophytes were distributed in numerous nodes of the phylogenetic tree of higher plants, displaying that halophytes evolved diverse mechanisms against high soil salinity (Flowers et al. 2010). This diversity complicates discovery of the salinity or alkali tolerance mechanisms of halophytes. Although research on halophytes is increasing (Flowers and Yeo 1995, Wang et al. 2007a,b; Ardie et al. 2009, 2010, 2011; Liu et al. 2009, Flowers et al. 2010, Yu et al. 2011, Yu et al. 2013, Zhang et al. 2013, Wang et al. 2017, Zhang et al. 2017), genome sequence of extreme halophytes was missing, which greatly restricts the study on salinity tolerance and alkali tolerance of extreme halophytes.

Leymus chinensis is constructive species in alkalized grassland in northern China (Zheng and Li 1999), with high forage value. L. chinensis can survive for a long time in alkaline grassland with $\mathrm{pH}$ above 10 (Zheng and Li 1999). It is one of the most alkali-tolerant Gramineae halophyte (Liu et al. 2015), and it also is close relative of wheat plants. In northern China, L. chinensis is an important forage grass that is used to improve alkaline grassland (Zheng and Li 1999). Understanding L. chinensis alkali tolerance would improve the current knowledge of alkali tolerance, and even could provide breeders with candidate alkali tolerance genes. Although some studies on physiological response (Liu et al. 2015, Wang et al. 2015), microRNAs (Zhai et al. 2014), and gene expression profiling (microarray chips) (Jin et al. 2008) had been carried out in L. chinensis, these studies only referred to the genome sequence of other species, which inevitably led to imperfection of the gene expression profiling. In this study, we used PacBio platform to constitute a reference full-length transcript sequences (45,037 highquality isoforms) for alkali-stressed L. chinensis, and we subsequently conducted a RNAseq analysis of alkali stress response by mapping Illumina reads to the generated reference transcript sequences. This approach will improve understanding of gene expression regulation of alkali-stress response in this species. Additionally, we also conducted biochemical and anatomical analysis. We aimed to elucidate the response mechanisms of L. chinensis to alkali stress through connecting biochemical analysis and transcriptomic profiling.

\section{Materials and methods}

Plant materials: Leymus chinensis is a perennial clone grass, sexual propagation (seed reproduction) is rare because of its extreme low seed germination rate, and vegetative reproduction is its dominant reproduction type. To minimize the effects of plant to plant, we used different ramets derived from one clone of L. chinensis as experimental materials. L. chinensis is not an endangered or protected species, therefore, no specific permissions were required for the plant collection. We transferred the $L$. chinensis individual (named as LC1 plant) from alkalized grassland located in northeast China to a greenhouse. First, we collected partial leaves and roots samples of $\mathrm{LC} 1$ plant and stored these samples at $-80^{\circ} \mathrm{C}$. After this, in order to obtain more rhizomes, we immediately divided the LC1 plant into several ramets, and then these ramets were grown in different plastic pots containing thoroughly washed sand and placed in a greenhouse $\left[24-26^{\circ} \mathrm{C}\right.$ (day) and $17-19^{\circ} \mathrm{C}$ (night) temperatures under 16-h light]. The ramets were watered daily with a Hoagland nutrient solution for $60 \mathrm{~d}$. After this, we collected all rhizomes from the ramets, and then the rhizomes were planted in different plastic pots containing thoroughly washed sand; each pot contained five rhizomes. The rhizomes were all generated form the LC1 plant, thus, belonged to a clone. These pots were watered daily with a Hoagland nutrient solution for $30 \mathrm{~d}$. After this, we selected pots with uniform ramets in order to perform further experiments.

Stress treatment for RNAseq, qPCR, physiological experiments, and anatomical analysis: Above mentioned pots (15) were used as control group, and another 15 pots were used as alkali-stress treatment group. Control group was watered with Hoagland nutrient solution, and alkali-stress treatment group was watered with alkaline salt solution that contained the same nutrient composition of Hoagland nutrient solution. Two alkaline salts were mixed in a 9:1 molar ratio $\left(\mathrm{NaHCO}_{3}\right.$ to $\left.\mathrm{Na}_{2} \mathrm{CO}_{3}, \mathrm{pH} 8.8\right)$ as the alkaline-stress treatment, and total salinity was $200 \mathrm{mM}$. The stress treatment duration was 2-30 d. All rhizomes used in this work were generated from the same individual through vegetative reproduction without meiosis and fertilization. Therefore, in theory, these ramets were genetically identical, which would improve accuracy of comparative transcriptome analysis. When the seedlings were exposed to alkali-stress treatment for $2 \mathrm{~d}$, we collected the leaf or root samples for biochemical measurements, RNA sequencing experiment, and realtime PCR analysis. When the seedlings were exposed to alkali-stress treatment for $30 \mathrm{~d}$, we prepared the leaf or root samples for biomass, chlorophyll (Chl), photosynthesis, chloroplast ultrastructure, and anatomical analyses. Leaves or roots of five seedlings (ramets) from one pot for each treatment were pooled as a biological replicate, with three biological replicates for each treatment.

Stress treatment for PacBio sequencing: The ramets used in PacBio sequencing experiment and the ramets used in RNA sequencing experiments were both generated from the LC1 plant, and belonged to the clone. First, we exposed the ramets to $300 \mathrm{mM} \mathrm{NaCl}$ and $200 \mathrm{mM}$ alkalistress treatment $\left(\mathrm{NaHCO}_{3}\right.$ to $\mathrm{Na}_{2} \mathrm{CO}_{3}, \mathrm{pH}$ 8.8) for 2 and $30 \mathrm{~d}$ through using the method described above. We collected the leaf, root, bud, spike, and flower tissues under three treatment conditions at the tillering, booting, and anthesis stage. Additionally, we also collected partial leaf and root samples of the initial LC1 plant for PacBio sequencing.

Chl and photosynthesis measurements: When the seedlings were exposed to alkali stress treatment for $30 \mathrm{~d}$, 
photosynthesis measurements were conducted. Net photosynthetic rate $\left(P_{\mathrm{N}}\right)$, stomatal conductance $\left(g_{\mathrm{s}}\right)$, and transpiration rate $(E)$ of leaves were determined using a portable open flow gas-exchange system LI-6400 (LICOR, USA) with PAR of $1,200 \mu \mathrm{mol}\left(\right.$ photon) $\mathrm{m}^{-2} \mathrm{~s}^{-1}$, leaf temperature of $28^{\circ} \mathrm{C}$, and vapor pressure deficit (VPD) of 2.4-2.8. Chl $a$, $\mathrm{Chl} b$, and carotenoids were extracted with $80 \%$ acetone, and absorbance of extracted solution was determined at 440, 645, and $663 \mathrm{~nm}$ with a spectrophotometer (T600, PERSEE, China). The calculation used the methods of Zhu (1993).

Biochemical measurement: When the seedlings were exposed to alkali stress treatment for $2 \mathrm{~d}$, leaves and roots were harvested and freeze-dried for biochemical measurement. Roots or leaves of five seedlings (ramets) for each treatment were pooled as a biological replicate, with three biological replicates for each treatment. Concentrations of free amino acids and sugars were determined with the methods of Zhao et al. (2017). Briefly, the free amino acids and sugars were isolated from freezedried samples using distilled water at $50^{\circ} \mathrm{C}$, and were further treated and loaded into a liquid chromatographytandem mass spectrometry system equipped with a highperformance liquid chromatograph (HPLC) and a triple quadrupole mass spectrometer (API3200MD, AB SCIEX). Dried samples were digested in $65 \% \mathrm{HNO}_{3}$ at $120^{\circ} \mathrm{C}$, and the $\mathrm{Na}^{+}$and $\mathrm{K}^{+}$contents were measured by an atomic absorption spectrophotometer (TAS-990super, PERSEE, China).

Anatomical analysis and chloroplast ultrastructure: When the seedlings were exposed to alkali-stress treatment for $30 \mathrm{~d}$, anatomical analysis and chloroplast ultrastructure experiments were conducted. The samples were fixed with FAA solution (10:50:5:35 - formaldehyde:ethanol:acetic acid:water), and then fixed samples were cleaned using $0.1 \mathrm{M}$ phosphate buffer ( $\mathrm{pH}$ 7.4). The leaf samples for chloroplast ultrastructure were fixed with $2.5 \%$ glutaraldehyde at $4^{\circ} \mathrm{C}$ for $6 \mathrm{~h}$, and were then rinsed with $0.1 \mathrm{M}$ phosphate buffer ( $\mathrm{pH}$ 7.4) three times. Then fixed samples for both experiments were further treated by $1 \% \mathrm{OsO}_{4}$ in $0.1 \mathrm{M}$ phosphate buffer $(\mathrm{pH} 7.4)$. The samples were dehydrated using ethanol and acetone in different concentration gradients. The samples were infiltrated in a solution of $1: 1$ acetone:embedding agent (EMBed 812$)$ for $3 \mathrm{~h}$, in 2:1 acetone:EMBed 812 overnight, and then pure EMBed 812 for $8 \mathrm{~h}$, before the samples were kept at $60^{\circ} \mathrm{C}$ for $48 \mathrm{~h}$. The embedded materials were sliced to $1-2 \mu \mathrm{m}$ thicknesses with an ultramicrotome (Leica UC7, Leica), and were dyed with toluidine blue, then photographs were taken by a scanner (3D HISTECH, Hungary). The embedded material was also sliced to $70-\mathrm{nm}$ ultrathin sections, and then the sections were stained with uranyl acetate for $15 \mathrm{~min}$, following $15 \mathrm{~min}$ staining with lead citrate. The chloroplast ultrastructure was observed under a transmission electron microscope (HT7700, Hitachi, Japan).

Reference full-length transcript sequence: We used PacBio Sequel platform (third generation sequencing technology) to produce reference full-length transcript sequen- ces for reference of mapping analysis of the RNAseq data generated by Illumina platform. We mixed all collected RNA samples in equal concentration of RNA, and then this mixed sample was exposed to PacBio sequencing (40 Gb). Finally, we obtained full-length sequences for all expressed transcript. PacBio sequence data were processed using the SMRTlink software. Circular consensus sequence (CCS) data was generated from subreads file (default parameters). Then the CCS BAM files were used to produce full-length transcript through using isoseq 3 software (default parameters). Function of the nonredundant transcripts was annotated against following databases: NR (NCBI nonredundant protein sequences), NT (NCBI nonredundant nucleotide sequences), Pfam (Protein family, protein domain), KOG/COG (Clusters of Orthologous Groups of proteins), SwissProt (a manually annotated and reviewed protein sequence database), GO (Gene Ontology), and KO (KEGG Ortholog database). We used the TransDecoder software to identify candidate protein-coding regions of transcript on basis of the prediction of an ORF (Open Reading Frame).

RNA sequencing: When the seedlings were exposed to alkali-stress treatment for $2 \mathrm{~d}$, we used conventional method to preform RNA-sequencing experiment. Leaves or roots of five seedlings from one pot for each treatment were pooled as a biological replicate, with three biological replicates for each treatment. Two $\mu \mathrm{g}$ RNA of each sample were used for the RNA input. The libraries were generated using NEBNext ${ }^{\circledR}$ Ultra $^{\mathrm{TM}}$ RNA Library Prep Kit for Illumina ${ }^{\circledR}$ (\#E7530L, Neb, USA). The Libraries were sequenced on Illumina NovaSeq 6000 and 150 bp pairedend reads were generated. Finally, about $10 \mathrm{~Gb}$ clean data for each sample were produced. Clean data were then aligned to reference full-length transcript sequences using HISAT2 v2.1.0. RSEM software was used to calculate FPKM (Reads Per Kilobase Millon Mapped Reads) of all nonredundant transcripts. We used DESeq 2 to identify the differentially expressed genes (DEGs) between control and stress treatments (adjusted $P$ value $\leq 0.05$ and $\mid \log 2$ fold change $\mid \geq 1$ ). The $P$-values were corrected by the BH method. DEGs were exposed to KEGG (Kyoto Encyclopedia of Genes and Genomes, http://www.kegg.jp/) enrichment by the hypergeometric test, in which $P$-values were adjusted by multiple comparisons as q-value.

Quantitative real-time PCR analysis: When the seedlings were exposed to alkali-stress treatment for $2 \mathrm{~d}$, we used conventional method to preform real-time PCR analysis. Roots of five seedlings (ramets) for each treatment were pooled as a biological replicate, with three biological replicates for each treatment. The total RNA from each sample was isolated with TRIzol reagent (Invitrogen). The RNA was treated with DNaseI (Invitrogen), reversetranscribed using SuperScriptTM RNase H-Reverse Transcriptase (Invitrogen), and then subjected to realtime PCR analysis. Amplification of the target gene was monitored every cycle by SYBR Green. Amplification of the actin gene (ID: Gene.42270) was used as an internal quantitative control. The primer sequences of actin gene were 5'-TACACGAAGCGACATACAATTCCATCA-3' 
(forward sequence) and 5'-AGAACCTCCACTGAGAACAACATTACC-3' (reverse sequence). The relative expression of the target genes was calculated using the $\triangle \triangle \mathrm{Ct}$ method (Livak and Schmittgen 2001).

Statistical analysis and experimental design: The experimental design was randomized complete block design. Physiological measurements, RNA sequencing experiment, and qRT-PCR experiment all included three biological replicates. Leaves or roots of five seedlings (ramets) from one pot for each treatment were pooled as a biological replicate. The statistical significance of physiological measurements and qRT-PCR were determined by the $t$-test at 0.05 level using SPSS 16.0 (IBM, USA). The gene expression data of RNA sequencing were analysed by $D E S e q 2 R$ package. The $P$-values generated in the RNA sequencing analysis were adjusted by the BH method. DEGs between control and stress treatments were defined as adjusted $P$ value $\leq 0.05$ and $\mid \log 2$ fold change $\mid \geq 1$.

\section{Results}

Physiological and anatomical response: Alkali stress markedly limited the photosynthesis and growth of L. chinensis. Alkali stress decreased the net photosynthetic rate $\left(P_{\mathrm{N}}\right)$, stomatal conductance $\left(g_{\mathrm{s}}\right)$, and transpiration rate $(E)$ of $L$. chinensis (Table 1). However, alkali stress did not affect accumulation of photosynthetic pigments (Table 1). Alkali stress decreased the biomass of root and leaf, but only produced small effect on leaf water content (Table 1). Alkali stress increased the $\mathrm{Na}^{+}$concentration and decreased $\mathrm{K}^{+}$concentration in both roots and leaves (Table 1). Alkali

Table 1. Effects of alkali stress on growth, photosynthesis, and ion contents in Leymus chinensis. The 30-d-old seedlings were exposed to alkali stress $\left(\mathrm{NaHCO}_{3}: \mathrm{Na}_{2} \mathrm{CO}_{3}, 9: 1 ; 200 \mathrm{mM}, \mathrm{pH} 8.8\right)$ for $30 \mathrm{~d}$. Values are means $( \pm \mathrm{SD})$ of three replicates. Water content $=($ fresh mass - dry mass $) \times 100 /$ fresh mass. Asterisk in alkali stress treatment column indicates significant difference between control and alkali stress conditions within the same tissue at 0.05 level ( $t$-test). NS - no significant difference; $P_{\mathrm{N}}-$ net photosynthetic rate; $g_{\mathrm{s}}$ - stomatal conductance; $E$ - transpiration rate; FM - fresh mass; DM - dry mass.

\begin{tabular}{lll}
\hline & Control & Alkali stress \\
\hline$P_{\mathrm{N}}\left[\mu \mathrm{mol}\left(\mathrm{CO}_{2}\right) \mathrm{m}^{-2} \mathrm{~s}^{-1}\right]$ & $19.56 \pm 0.87$ & $10.88 \pm 1.09^{*}$ \\
$g_{\mathrm{s}}\left[\mathrm{mol}\left(\mathrm{H}_{2} \mathrm{O}\right) \mathrm{m}^{-2} \mathrm{~s}^{-1}\right]$ & $0.33 \pm 0.04$ & $0.09 \pm 0.01^{*}$ \\
$E\left[\mathrm{mmol}\left(\mathrm{H}_{2} \mathrm{O}\right) \mathrm{m}^{-2} \mathrm{~s}^{-1}\right]$ & $7.43 \pm 0.58$ & $2.49 \pm 0.14^{*}$ \\
Chlorophyll $a\left[\mathrm{mg} \mathrm{g}^{-1}(\mathrm{FM})\right]$ & $0.84 \pm 0.06$ & $0.80 \pm 0.18^{\mathrm{NS}}$ \\
Chlorophyll $b\left[\mathrm{mg} \mathrm{g}^{-1}(\mathrm{FM})\right]$ & $0.46 \pm 0.02$ & $0.39 \pm 0.07^{\mathrm{NS}}$ \\
Carotenoid $\left[\mathrm{mg} \mathrm{g}^{-1}(\mathrm{FM})\right]$ & $0.34 \pm 0.02$ & $0.30 \pm 0.05^{\mathrm{NS}}$ \\
Leaf dry mass $[\mathrm{g}$ per plant $]$ & $2.53 \pm 0.11$ & $1.28 \pm 0.08^{*}$ \\
Root dry mass $[\mathrm{g}$ per plant $]$ & $0.56 \pm 0.06$ & $0.37 \pm 0.05^{*}$ \\
Leaf water content $[\%]$ & $72.0 \pm 5.3$ & $63.0 \pm 0.4^{\mathrm{NS}}$ \\
Leaf $\mathrm{Na}^{+}\left[\mu \mathrm{mol} \mathrm{g}{ }^{-1}(\mathrm{DM})\right]$ & $53.8 \pm 9.4$ & $321.9 \pm 58.6^{*}$ \\
Root $\mathrm{Na}^{+}[\mu \mathrm{mol} \mathrm{g}(\mathrm{DM})]$ & $60.6 \pm 28.3$ & $475.4 \pm 28.2^{*}$ \\
Leaf $\mathrm{K}^{+}\left[\mu \mathrm{mol} \mathrm{g}{ }^{-1}(\mathrm{DM})\right]$ & $747.5 \pm 17.4$ & $509.1 \pm 8.9^{*}$ \\
Root $\mathrm{K}^{+}\left[\mu \mathrm{mol} \mathrm{g}{ }^{-1}(\mathrm{DM})\right]$ & $483.7 \pm 53.7$ & $140.5 \pm 3.1^{*}$ \\
\hline
\end{tabular}

stress affected only marginally the anatomical structure of leaf (Fig. 1). Alkali stress slightly reduced the vessel diameter of the major vein and aerenchyma volume (Fig. 1). Alkali stress increased packing density of thylakoids in chloroplasts, and the chloroplast of control plant (48 grana per chloroplast) had more grana than that of stressed plant (19 grana per chloroplast) (Fig. 2). We detected 19 amino acids in both roots and leaves of L. chinensis (Table 2). Alkali stress increased only the concentration of proline in roots and the concentration of asparagine in leaves (Table 2). Accumulation of most carbohydrates was stimulated by alkali stress in leaves, while only concentrations of fructose, glucose, mannose, sucrose, and maltose were enhanced in roots (Table 2). Among four polyols, accumulation of sorbitol/mannitol was stimulated by alkali stress in roots. In stressed leaves, contributions of fructose $(9.92 \%)$ and sucrose $(33.34 \%)$ to total molarity were greater than those of other solutes, and they played an important role in osmotic adjustment (Table 2). In

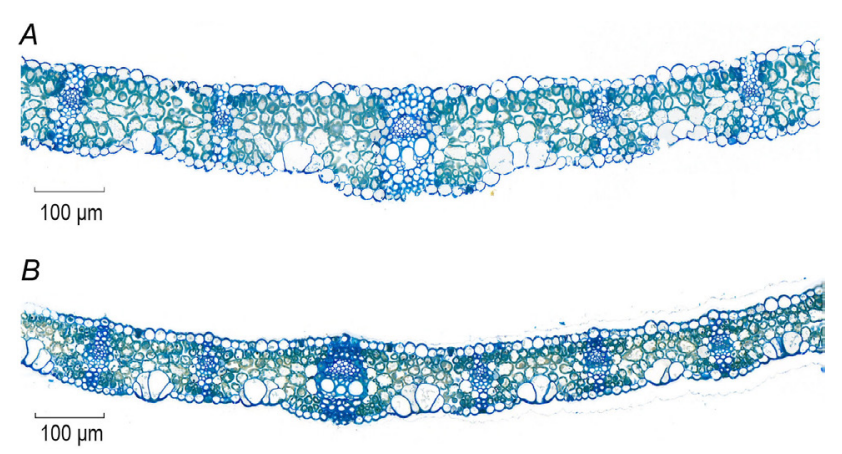

Fig. 1. Effects of alkali stress on leaf anatomy of Leymus chinensis. $(A)$ control leaf and $(B)$ stressed leaf. The 30-d-old seedlings were exposed to alkali stress $\left(\mathrm{NaHCO}_{3}: \mathrm{Na}_{2} \mathrm{CO}_{3}, 9: 1\right.$; $200 \mathrm{mM}$, pH 8.8) for $30 \mathrm{~d}$.

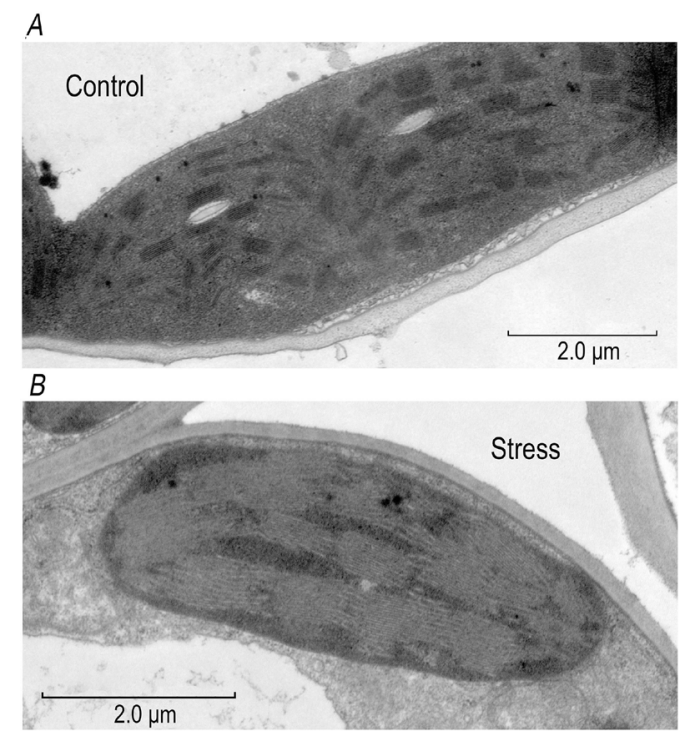

Fig. 2. Effects of alkali stress on chloroplast ultrastructure in Leymus chinensis. The 30-d-old seedlings were exposed to alkali stress $\left(\mathrm{NaHCO}_{3}: \mathrm{Na}_{2} \mathrm{CO}_{3}, 9: 1 ; 200 \mathrm{mM}, \mathrm{pH} 8.8\right)$ for $30 \mathrm{~d}$. 
Table 2. Fold change and percent contribution to total molarity of each compatible solute in Leymus chinensis. Fold change is ratio of stress and control. The 30-d-old seedlings were exposed to alkali stress $\left(\mathrm{NaHCO}_{3}: \mathrm{Na}_{2} \mathrm{CO}_{3}, 9: 1 ; 200 \mathrm{mM}, \mathrm{pH}\right.$ 8.8) for $2 \mathrm{~d}$. Five seedlings (ramets) from one pot for each treatment were pooled as a biological replicate, with three biological replicates for each treatment. CL control leaf; SL - stressed leaf; CR - control root; SR - stressed root. Percent contribution is calculated with following equation: percent contribution of a given solute $=$ its molarity concentration $\times 100 /$ total molarity concentration, where total molarity concentration is sum of molarity concentrations of all detected solutes. ${ }^{a}$ The solute was not detected in the alkali stressed plants. nd - not detected.

\begin{tabular}{|c|c|c|c|c|c|c|c|c|c|}
\hline & & \multicolumn{4}{|c|}{ Percent contribution to total molarity } & \multicolumn{2}{|l|}{ Leaf } & \multicolumn{2}{|l|}{ Root } \\
\hline & & $\mathrm{CL}$ & SL & $\mathrm{CR}$ & SR & Fold change & $P$ value & Fold change & $P$ value \\
\hline \multirow{19}{*}{$\begin{array}{l}\text { Free amino } \\
\text { acids }\end{array}$} & Glycine & 1.58 & 2.46 & 0.85 & 0.42 & 3.42 & 0.490 & 0.68 & 0.056 \\
\hline & Alanine & 17.84 & 5.27 & 7.31 & 3.74 & 0.65 & 0.307 & 0.71 & 0.118 \\
\hline & Serine & 5.12 & 5.52 & 3.43 & 1.22 & 2.37 & 0.498 & 0.49 & 0.001 \\
\hline & Proline & 2.49 & 1.54 & 0.41 & 1.19 & 1.36 & 0.462 & 3.99 & 0.000 \\
\hline & Valine & 3.16 & 1.03 & 2.99 & 0.70 & 0.72 & 0.628 & 0.33 & 0.000 \\
\hline & Threonine & 3.30 & 1.77 & 3.17 & 0.96 & 1.18 & 0.804 & 0.42 & 0.001 \\
\hline & Cysteine & 1.15 & 0.69 & 0.69 & 0.30 & 1.32 & 0.658 & 0.60 & 0.058 \\
\hline & Isoleucine & 1.60 & 0.54 & 1.22 & 0.30 & 0.74 & 0.587 & 0.33 & 0.000 \\
\hline & Asparagine & 4.46 & 5.64 & 29.50 & 19.08 & 2.79 & 0.036 & 0.89 & 0.606 \\
\hline & Aspartic acid & 6.54 & 3.83 & 5.91 & 6.07 & 1.29 & 0.575 & 1.42 & 0.005 \\
\hline & Glutamine & 0.00 & 0.46 & 1.64 & 0.74 & ${ }^{\mathrm{a}} 100$ & 0.367 & 0.62 & 0.016 \\
\hline & Glutamic acid & 2.22 & 1.72 & 3.84 & 4.13 & 1.70 & 0.444 & 1.49 & 0.006 \\
\hline & Histidine & 0.80 & 1.30 & 0.77 & 0.32 & 3.58 & 0.462 & 0.57 & 0.001 \\
\hline & Phenylalanine & 1.54 & 0.45 & 0.47 & 0.14 & 0.65 & 0.358 & 0.42 & 0.003 \\
\hline & Arginine & 2.70 & 2.27 & 2.96 & 2.16 & 1.85 & 0.409 & 1.01 & 0.878 \\
\hline & Tryptophan & 0.34 & 0.17 & 0.41 & 0.23 & 1.10 & 0.907 & 0.79 & 0.205 \\
\hline & Lysine & 4.82 & 1.87 & 2.82 & 0.82 & 0.85 & 0.809 & 0.40 & 0.001 \\
\hline & Tyrosine & 1.95 & 0.66 & 0.59 & 0.19 & 0.75 & 0.596 & 0.44 & 0.007 \\
\hline & Leucine & 3.30 & 0.95 & 1.92 & 0.40 & 0.63 & 0.333 & 0.28 & 0.000 \\
\hline \multirow{10}{*}{$\begin{array}{l}\text { Free } \\
\text { carbohydrates }\end{array}$} & Erythrose & 4.41 & 3.47 & 6.52 & 5.85 & 1.73 & 0.063 & 1.24 & 0.047 \\
\hline & Fructose & 3.48 & 9.92 & 1.96 & 3.98 & 6.29 & 0.000 & 2.81 & 0.001 \\
\hline & Xylose & 0.04 & 0.01 & 0.25 & 0.17 & 0.88 & 0.935 & 0.95 & 0.851 \\
\hline & Glucose & 0.17 & 0.35 & 0.11 & 0.33 & 4.67 & 0.048 & 3.94 & 0.006 \\
\hline & Galactose & 0.02 & 0.04 & 0.00 & 0.00 & 3.64 & 0.276 & nd & nd \\
\hline & Mannose & 2.29 & 1.81 & 1.30 & 1.47 & 1.74 & 0.009 & 1.56 & 0.006 \\
\hline & Sucrose & 11.63 & 33.34 & 9.52 & 37.20 & 6.32 & 0.000 & 5.40 & 0.000 \\
\hline & Ribose & 0.56 & 1.12 & 0.55 & 0.27 & 4.39 & 0.005 & 0.67 & 0.239 \\
\hline & Maltose & 0.78 & 0.48 & 0.43 & 0.52 & 1.35 & 0.008 & 1.68 & 0.027 \\
\hline & Trehalose & 0.01 & 0.04 & 0.01 & 0.02 & 11.79 & 0.035 & 2.24 & 0.560 \\
\hline \multirow[t]{4}{*}{ Polyols } & Sorbitol/mannitol & 0.50 & 0.15 & 0.05 & 0.09 & 0.68 & 0.132 & 2.76 & 0.015 \\
\hline & Pinitol & 9.42 & 10.50 & 7.63 & 6.73 & 2.46 & 0.287 & 1.22 & 0.789 \\
\hline & Xylitol & 0.16 & 0.08 & 0.00 & 0.00 & 1.11 & 0.608 & nd & nd \\
\hline & Inositol & 1.61 & 0.56 & 0.78 & 0.27 & 0.77 & 0.092 & 0.48 & 0.009 \\
\hline
\end{tabular}

stressed roots, sucrose (37.2\%) and asparagine (19.08\%) exhibited much higher contributions to osmotic potential (total molarity concentration) than other solutes (Table 2).

Reference full-length transcript sequence: We used PacBio platform to constitute a reference full-length transcript sequence (Table 1S, supplement). Finally, we obtained 45,037 high-quality isoforms (Table $2 \mathrm{~S}$, supplement).

Transcriptomic profiling: All gene expression data were showed in Tables 3S, 4S (supplement). We found that 2,216 genes were differentially expressed under control and stress conditions in the leaves, including 693 upregulated genes and 1,523 downregulated genes. We observed that 1,417 genes were differentially expressed under control and stress conditions in the roots, including 721 upregulated genes and 696 downregulated genes. We exposed all differentially expressed genes (DEGs) to KEGG enrichment. DEGs were significantly enriched in nine pathways in leaves, including antenna proteins, photosynthesis, glycine, serine, and threonine metabolism, glyoxylate and dicarboxylate metabolism, gaffeine metabolism, fatty acid elongation, phenylpropanoid biosynthesis, ribosome biogenesis, and ubiquinone and 
other terpenoid-quinone biosynthesis. In the leaves, four pathways (antenna proteins, photosynthesis, glycine, serine and threonine metabolism, and glyoxylate and dicarboxylate metabolism) were significantly restricted by alkali stress, and ribosome biogenesis was significantly promoted by alkali stress (Fig. 3; Table 5S, supplement). In KEGG pathway network, photosynthesis pathway is composed of all photosynthetic electron transport protein genes. Key photosynthetic electron transport protein genes (21) and 32 antenna protein genes were downregulated in leaves under alkali stress (Table 5S). In roots, DEGs were significantly enriched in ten pathways, including phenylpropanoid biosynthesis, sesquiterpenoid and triterpenoid biosynthesis, nitrogen metabolism, isoquinoline alkaloid biosynthesis, alanine, aspartate and glutamate metabolism, sulfur metabolism, tropane, piperidine and pyridine alkaloid biosynthesis, amino sugar and nucleotide sugar metabolism, galactose metabolism, and phenylalanine metabolism (Fig. 4). It was obvious that nitrogen metabolism was significantly upregulated in the roots (Fig. 4).

We also identified many differentially expressed salinity-tolerant genes (Table 3). For example, three $N H X$ genes were significantly upregulated in roots under alkali stress, and one $N H X$ gene was significantly upregulated in leaves (Table 3). Three late embryogenesis abundant (LEA) genes and one dehydrin gene were significantly upregulated in roots under alkali stress, while the four genes all were downregulated in the leaves. One potassium transporter gene was significantly upregulated in leaves but not in roots. Both HKT4 and HKT8 were downregulated in roots. $\mathrm{V}-\mathrm{H}^{+}$-ATPase also was downregulated in leaves, but HKT8 was upregulated in leaves (Table 3). Six highaffinity nitrate transporter (NRT) genes, one glutamate dehydrogenase $(G D H)$ gene, and one glutamine synthetase $(G S 1 ; 3)$ gene were upregulated in roots (Table 4). Most of NRT1/PTR FAMILY (NPF) genes was downregulated in both roots and leaves (Table 4). Many glutathione $S$-transferase and peroxidase genes were expressed in stressed leaves or roots, but the most of these expressed genes were downregulated in roots or leaves (Table 6S, supplement).

Validation of qPCR: The results of RNAseq were validated by qRT-PCR (Table 7S, supplement). Eight out of 11 genes tested showed consistent results between RNAseq and qRT-PCR (Table 7S), indicating the results of RNAseq were reliable.

\section{Discussion}

Growth and photosynthesis: In the leaves of L. chinensis, many genes involved in photosynthetic light reaction were downregulated by alkali stress (Fig. 3, Table 5S). This might be an adaptive strategy of L. chinensis to alkali stress. Alkali stress may promote the shift of the energy from biomass accumulation to stress response by restricting synthesis of proteins involved in building plant body (Munns and Gilliham 2015). Under alkali stress, growth of L. chinensis was slowed or even stopped (Table 1), which may be mediated through downregulation of photosynthetic electron transport protein genes. Accordingly, we observed a reduction in $P_{\mathrm{N}}, g_{\mathrm{s}}$, and $E$ in $L$. chinensis under alkali stress. Surprisingly, alkali stress did not influence photosynthetic pigment content of L. chinensis, which was not consistent with finding in wheat plants where alkali stress caused $\mathrm{Na}^{+}$excess and destroyed pigments in chloroplasts (Yang et al. 2008b). Higher stability of photosynthetic apparatus under alkali stress may be important alkali-tolerance trait of $L$. chinensis. $P_{\mathrm{N}}$ is determined by photosynthetic electron transport and carbon assimilation rate. Under alkali stress, decreased $P_{\mathrm{N}}$ of $L$. chinensis may be due to decreased photosynthetic electron transport rate (Fig. 3, Table 5S) and lower $g_{\mathrm{s}}$ (Table 1). Under alkali stress, reduced aerenchyma volume may also influence the $\mathrm{CO}_{2}$ influx into mesophyll cells of L. chinensis (Fig. 1). In addition, ribosome biogenesis process was significantly promoted by alkali stress in the leaves of L. chinensis (Fig. 3). Under alkali stress,

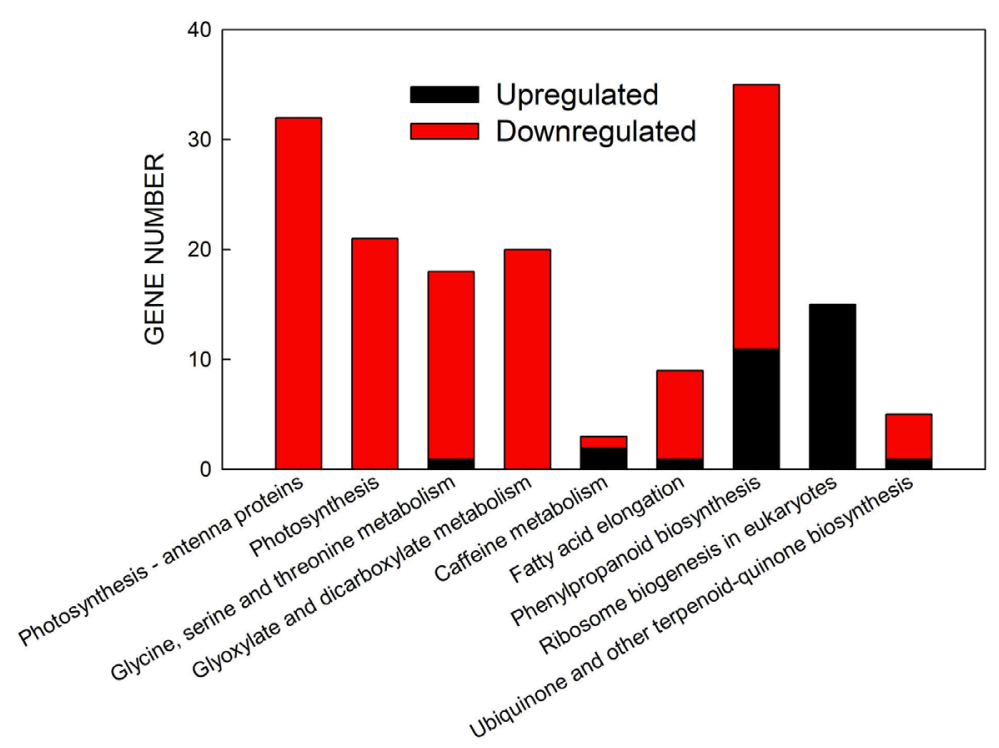

Fig. 3. Kyoto Encyclopedia of Genes and Genomes (KEGG) enrichment of differentially expressed genes in leaves of Leymus chinensis. Five seedlings (ramets) from one pot for each treatment were pooled as a biological replicate, with three biological replicates for each treatment. The 30 -d-old seedlings were exposed to alkali stress $\left(\mathrm{NaHCO}_{3}: \mathrm{Na}_{2} \mathrm{CO}_{3}\right.$, 9:1; $200 \mathrm{mM}, \mathrm{pH} 8.8$ ) for $2 \mathrm{~d}$. KEGG pathways with adjusted $P<0.05$ are displayed. 


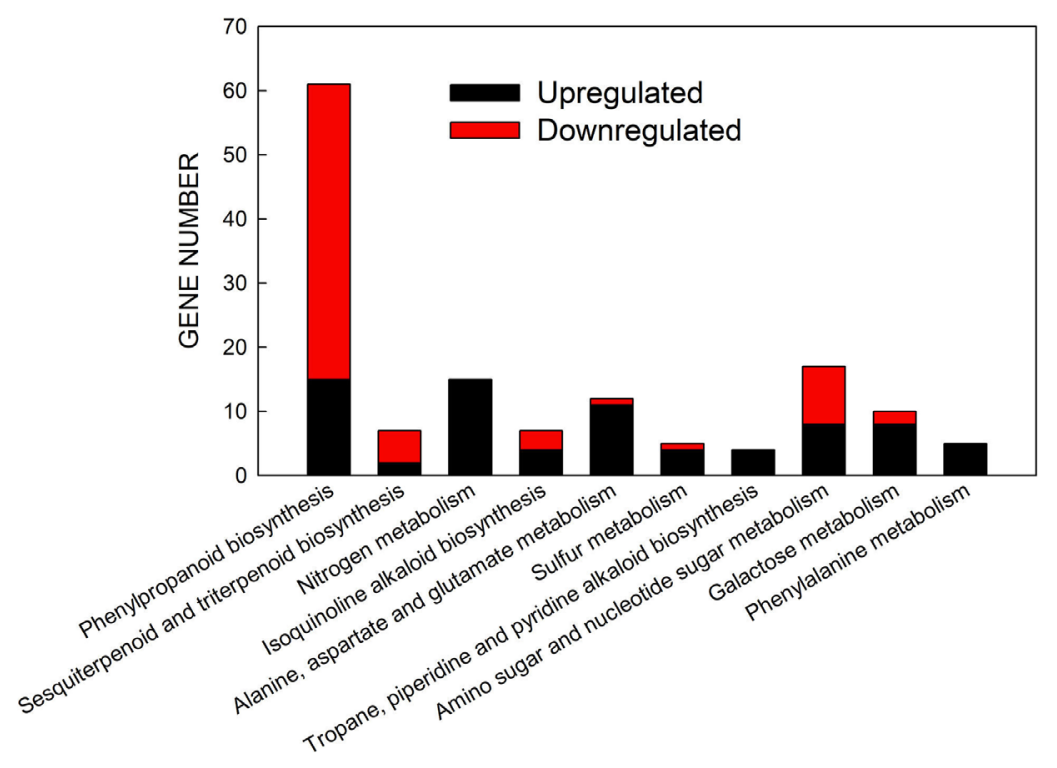

Fig. 4. Kyoto Encyclopedia of Genes and Genomes (KEGG) enrichment of differentially expressed genes in roots of Leymus chinensis. Five seedlings (ramets) from one pot for each treatment were pooled as a biological replicate, with three biological replicates for each treatment. The 30-d-old seedlings were exposed to alkali stress $\left(\mathrm{NaHCO}_{3}: \mathrm{Na}_{2} \mathrm{CO}_{3}, 9: 1 ; 200 \mathrm{mM}\right.$, $\mathrm{pH} 8.8$ ) for $2 \mathrm{~d}$. KEGG pathways with adjusted $P<0.05$ are displayed.

Table 3. Effects of alkali stress on gene expression involved in osmotic adjustment and ion homeostasis in Leymus chinensis. Five seedlings (ramets) from one pot for each treatment were pooled as a biological replicate, with three biological replicates for each treatment. The 30-d-old seedlings were exposed to alkali stress $\left(\mathrm{NaHCO}_{3}: \mathrm{Na}_{2} \mathrm{CO}_{3}, 9: 1 ; 200 \mathrm{mM}, \mathrm{pH} 8.8\right)$ for $2 \mathrm{~d}$. NCL - sodium/ calcium exchanger; NHX - sodium/hydrogen exchanger; LEA - late embryogenesis abundant protein; HKT - high-affinity potassium transporter.

\begin{tabular}{llllll}
\hline & & \multicolumn{2}{l}{ Root } & \multicolumn{2}{l}{ Leaf } \\
Gene ID & Gene name & Fold change & Adjusted $P$ value & Fold change & Adjusted $P$ value \\
\hline Gene.15906 & HKT4 & 0.49 & 0.001 & 0.80 & 0.358 \\
Gene.20677 & HKT8 & 0.24 & 0.000 & 25.35 & 0.167 \\
Gene.16840 & Ca ${ }^{2+} H^{+}$-exchanger & 0.45 & 0.000 & 0.99 & 0.986 \\
Gene.14224 & NCL1 & 0.42 & 0.000 & 0.63 & 0.001 \\
Gene.8886: & Potassium transporter & 0.47 & 0.007 & 0.50 & 0.000 \\
Gene.3756: & NHX & 1.40 & 0.039 & 3.08 & 0.000 \\
Gene.38145 & NHX & 4.89 & 0.000 & 1.66 & 0.083 \\
Gene.36084 & NHX & 2.14 & 0.000 & 1.45 & 0.079 \\
Gene.19378 & NHX & 3.42 & 0.001 & 1.06 & 0.965 \\
Gene.45017 & V-H+-ATPase & 0.59 & 0.017 & 0.17 & 0.002 \\
Gene.37596 & Potassium transporter & 1.27 & 0.316 & 2.75 & 0.045 \\
Gene.47912 & LEA_4 & 2.78 & 0.037 & 0.52 & 0.174 \\
Gene.33695 & Dehydrin & 8.20 & 0.001 & 0.02 & 0.000 \\
Gene.51088 & Dehydrin & 1.22 & 0.421 & 0.17 & 0.000 \\
Gene.33771 & LEA_4 & 0.59 & 0.430 & 0.38 & 0.020 \\
Gene.30351 & LEA_2 & 2.40 & 0.000 & 0.38 & 0.000 \\
Gene.46909 & LEA_2 & 3.42 & 0.030 & 0.58 & 0.482 \\
\hline
\end{tabular}

to support the biosynthesis of stress-response proteins, L. chinensis may generate more ribosome through enhanced gene expression involved in ribosome-biogenesis process. Alkali stress increased packing density of thylakoids in L. chinensis chloroplasts. Higher-density thylakoids may generate more ATP and NADPH to fuel alkali-stress responses.

Osmotic adjustment and ion homeostasis: High $\mathrm{K}^{+} / \mathrm{Na}^{+}$ ratios in cytoplasm are salinity-tolerance traits shared by most glycophytes and some halophytes (Wang and Xia 2018, Abdelraheem et al. 2019, Ganie et al. 2019). Under salinity stress, salinity-tolerant crops or halophytes compartmentalize $\mathrm{Na}^{+}$in vacuole in order to reduce its concentrations in cytoplasm. $\mathrm{Na}^{+}$compartmentation mechanism is conserved among higher plants, which is mediated by NHX (Flowers et al. 2019). To resist the osmotic stress due to high concentration of $\mathrm{Na}^{+}$or $\mathrm{Cl}^{-}$in vacuole, these plants also accumulate compatible solutes and $\mathrm{K}^{+}$in cytoplasm. Compatible solutes also function in 
Table 4. Effects of alkali stress on gene expression involved in nitrogen metabolism in Leymus chinensis. Five seedlings (ramets) from one pot for each treatment were pooled as a biological replicate, with three biological replicates for each treatment. The 30-d-old seedlings were exposed to alkali stress $\left(\mathrm{NaHCO}_{3}: \mathrm{Na}_{2} \mathrm{CO}_{3}, 9: 1 ; 200 \mathrm{mM}, \mathrm{pH}\right.$ 8.8) for $2 \mathrm{~d}$. GDH - glutamate dehydrogenase; $\mathrm{NRT}$ - highaffinity nitrate transporter; NPF - NRT1/PTR FAMILY; GS - glutamine synthetase.

\begin{tabular}{llllll}
\hline Gene ID & Gene name & $\begin{array}{l}\text { Root } \\
\text { Fold change }\end{array}$ & Adjusted $P$ value & $\begin{array}{l}\text { Leaf } \\
\text { Fold change }\end{array}$ & Adjusted $P$ value \\
\hline Gene.41379 & GDH2 & 2.464 & 0.000 & 0.798 & 0.711 \\
Gene.8701: & NRT2 & 3.043 & 0.000 & 0.542 & 0.329 \\
Gene.40163 & NRT2;1 & 25.460 & 0.000 & 0.193 & 0.691 \\
Gene.40014 & NRT2;2 & 13.442 & 0.000 & & \\
Gene.40106 & NRT2;2 & 12.648 & 0.000 & & \\
Gene.41118 & NRT2;2 & 7.759 & 0.000 & 2.624 & 1.000 \\
Gene.49732 & NRT2;1 & 2.628 & 0.000 & 0.345 & 0.802 \\
Gene.17652 & NPF2.3 & 0.392 & 0.000 & 0.569 & 0.058 \\
Gene.16130 & NPF5.2 & 0.271 & 0.000 & 0.698 & 0.003 \\
Gene.39847 & NPF6.2 & 0.080 & 0.158 & 0.199 & 0.000 \\
Gene.15644 & NPF6.2 & 0.095 & 1.000 & 0.471 & 0.009 \\
Gene.15751 & NPF6.2 & 0.187 & 0.166 & 0.261 & 0.000 \\
Gene.12799 & NPF6.3 & 0.454 & 0.000 & 0.203 & 0.000 \\
Gene.15464 & NPF2.11 & 0.170 & 0.000 & & \\
Gene.39536 & NPF8.3 & 0.098 & 0.008 & 1.436 & 0.000 \\
Gene.16031 & NPF8.5 & 1.343 & 1.000 & 0.246 & 0.001 \\
Gene.25764 & GS1;3 & 2.387 & 0.003 & 0.095 & 0.541 \\
\hline
\end{tabular}

prevention of protein aggregation in cytosol. Our results showed that, in L. chinensis, alkali stress enhanced the $\mathrm{Na}^{+}$concentration and decreased the $\mathrm{K}^{+}$concentration. In L. chinensis, accumulation of most carbohydrates was greatly stimulated under alkali stress, but accumulation of few free amino acids and polyols was stimulated (Table 2), indicating that carbohydrate may play more important role in osmotic adjustment and protein aggregation prevention than that of free amino acids and polyols.

Another common mechanism against $\mathrm{Na}^{+}$toxicity is the $\mathrm{Na}^{+}$exclusion from shoots into roots or from roots into rhizosphere solution. In rice and wheat, HKT1;5 (HKT8) gene mediates this $\mathrm{Na}^{+}$exclusion process (Wang and Xia 2018, Abdelraheem et al. 2019, Ganie et al. 2019). However, we found the HKT1;5 (HKT8) was significantly downregulated in roots of $L$. chinensis but upregulated in leaves (Table 3 ). We propose that $L$. chinensis may employ different transporter to exclude $\mathrm{Na}^{+}$from shoots into roots or from roots into rhizosphere solution. In addition, we observed upregulation of three $N H X$ genes in L. chinensis roots under alkali stress. This was consistent with finding of Wang et al. (2015) in rice plants, indicating that the $\mathrm{Na}^{+}$compartmentation mechanism under alkali stress is conserved between $L$. chinensis and glycophyte grass.

Dehydrin and LEA proteins are crucial protective proteins under salinity and osmotic stresses (Rorat et al. 2006). As dehydrin and LEA proteins contain tandem hydrophilic amino acids, they play important roles in the preventions of cytosol dehydration and protein aggregation (Rorat et al. 2006). LEA and dehydrin proteins also interact with carbohydrates to form intracellular glasses. The intracellular glasses can slow molecular mobility of water and ions (Buitink and Leprince 2008), which restricts the movement of $\mathrm{Na}^{+}$and $\mathrm{Cl}^{-}$in cytoplasm. Combination of enhanced carbohydrate concentration and upregulated $L E A$ and dehydrin expression would promote accumulation of intracellular glasses in L. chinensis leaves under alkali stress, alleviating the ion toxicity. Although we observed that many glutathione S-transferase and peroxidase genes were expressed in stressed L. chinensis plants, few of them were upregulated (Table 6S). This suggested that different members of antioxidant enzyme gene family may play different roles in detoxification of reactive oxygen, with tissue- or stress-type specificity.

Enhanced expression of nitrate transporter genes contributed to alkali tolerance of $\boldsymbol{L}$. chinensis: Plant roots absorb nitrate $\left(\mathrm{NO}_{3}^{-}\right)$by a large family of transporters that include NRT1/PTR FAMILY (NRT1) family and NRT2 family. $\mathrm{NO}_{3}{ }^{-}$absorbed by roots is further reduced to nitrite by nitrate reductase and then to $\mathrm{NH}_{4}^{+}$by nitrite reductase (NiR). $\mathrm{NH}_{4}^{+}$generated by nitrate reduction is assimilated by glutamine synthetase (GS) and glutamate synthase (GOGAT) or alternative glutamate dehydrogenase (GDH) pathway (Shi et al. 2009). Alkali stress strongly increased the gene expression level involved in nitrogen uptake and assimilation in rice plants, including $G D H$ genes, NRT2 genes, and GS genes (Wang et al. 2012). Similarly, we also observed that alkali stress mightily upregulated the expression of several NRT2 genes, GS1;3 gene, and GDH2 gene in L. chinensis. This displayed that the halophyte grass and glycophyte grass may share the $\mathrm{N}$ metabolism response mechanism under alkali stress. $\mathrm{NO}_{3}^{-}$uptake is mediated by a $\mathrm{H}^{+} / \mathrm{NO}_{3}{ }^{-}$symporter using the transmembrane proton 
gradient as driving force (Crawford and Glass 1998). Under alkali stress, the lack of external protons would weaken the $\mathrm{NO}_{3}{ }^{-}$uptake (Wang et al. 2012). Under alkali stress, L. chinensis might enhance the expression of NRT2 genes in roots to increase the frequency of $\mathrm{NO}_{3}^{-}$uptake.

Conclusions: L. chinensis shares many alkali tolerance mechanisms with rice or wheat plant. For example, under alkali stress, the $N H X$ expression response and nitrogen metabolism response mechanisms of $L$. chinensis were similar to those of rice plants. The apparent difference between $L$. chinensis and glycophyte in alkali-stress response was that photosynthetic pigments of L. chinensis were unaffected by long-term strong alkali stress ( $30 \mathrm{~d})$, but the pigment contents of wheat plants severely decreased under alkali stress. Compared to glycophytes, L. chinensis may have strong capacity to lower the toxicity of $\mathrm{Na}^{+}$to organelles and cytoplasmic proteins. Enhanced expression of dehydrin and LEA genes and increased accumulation of carbohydrates may contribute to the development of $\mathrm{Na}^{+}$-specific stress tolerance of $L$. chinensis under alkali stress.

\section{References}

Abdelraheem A., Esmaeili N., O'Connell M., Zhang J.: Progress and perspective on drought and salt stress tolerance in cotton. - Ind. Crop. Prod. 130: 118-129, 2019.

Ardie S.W., Liu S.K., Takano T.: Expression of the $A K T 1$-type $\mathrm{K}^{+}$ channel gene from Puccinellia tenuiflora, PutAKT1, enhances salt tolerance in Arabidopsis. - Plant Cell Rep. 29: 865-874, 2010.

Ardie S.W., Nishiuchi S., Liu S.K., Takano T.: Ectopic expression of the channel $\beta$ subunits from Puccinellia tenuiflora $(K P u t B 1)$ and rice $(K O B 1)$ alters $\mathrm{K}^{+} / \mathrm{Na}^{+}$homeostasis of yeast and Arabidopsis. - Mol. Biotechnol. 48: 76-86, 2011.

Ardie S.W., Xie L., Takahashi R. et al.: Cloning of a high-affinity $\mathrm{K}^{+}$transporter gene PutHKT2;1 from Puccinellia tenuiflora and its functional comparison with OsHKT2;1 from rice in yeast and Arabidopsis. - J. Exp. Bot. 60: 3491-3502, 2009.

Buitink J., Leprince O.: Intracellular glasses and seed survival in the dry state. - C. R. Biol. 331: 788-795, 2008.

Crawford N.M., Glass A.D.M.: Molecular and physiological aspects of nitrate uptake in plants. - Trends Plant Sci. 10: 389-395, 1998.

Flowers T.J, Yeo A.R.: Breeding for salinity resistance in crop plants: Where next? - Aust. J. Plant Physiol. 22: 875-884, 1995.

Flowers T.J., Galal H.K., Bromham L.: Evolution of halophytes: multiple origins of salt tolerance in land plants. - Funct. Plant Biol. 37: 604-612, 2010.

Flowers T.J., Glenn E.P., Volkov V.: Could vesicular transport of $\mathrm{Na}^{+}$and $\mathrm{Cl}^{-}$be a feature of salt tolerance in halophytes? Ann. Bot.-London 123: 1-18, 2019.

Ganie S.A., Molla K.A., Henry R.J. et al.: Advances in understanding salt tolerance in rice. - Theor. Appl. Genet. 132: 851-870, 2019.

Guo R., Shi L., Yang C. et al.: Comparison of ionomic and metabolites response under alkali stress in old and young leaves of cotton (Gossypium hirsutum L.) seedlings. - Front. Plant Sci. 7: 1785, 2016.

He R., Yu G., Han X. et al.: ThPP1 gene, encodes an inorganic pyrophosphatase in Thellungiella halophila, enhanced the tolerance of the transgenic rice to alkali stress. - Plant Cell Rep. 36: 1929-1942, 2017.

Jia X.M., Wang H., Sofkova S. et al.: Comparative physiological responses and adaptive strategies of apple Malus halliana to salt, alkali and saline-alkali stress. - Sci. Hortic.-Amsterdam 245:154-162, 2019.

Jin H., Kim H.R., Plaha P. et al.: Expression profiling of the genes induced by $\mathrm{Na}_{2} \mathrm{CO}_{3}$ and $\mathrm{NaCl}$ stresses in leaves and roots of Leymus chinensis. - Plant Sci. 175: 784-792, 2008.

Kaashyap M., Ford R., Bohra A. et al.: Improving salt tolerance of chickpea using modern genomics tools and molecular breeding. - Curr. Genomics 18: 557-567, 2017.

Li R., Shi F., Fukuda K.: Interactive effects of various salt and alkali stresses on growth, organic solutes, and cation accumulation in a halophyte Spartina alterniflora (Poaceae). Environ. Exp. Bot. 68: 66-74, 2010.

Liu B., Kang C., Wang X., Bao G.: Physiological and morphological responses of Leymus chinensis to saline-alkali stress. - Grassl. Sci. 61: 217-226, 2015.

Liu H., Zhang X.X., Takano T., Liu S.K.: Characterization of a PutCAX1 gene from Puccinellia tenuiflora that confers $\mathrm{Ca}^{2+}$ and $\mathrm{Ba}^{2+}$ tolerance in yeast. - Biochem. Bioph. Res. Co. 383: 392-396, 2009.

Livak K.J., Schmittgen T.D.: Analysis of relative gene expression data using real-time quantitative PCR and the $2^{-\Delta \Delta C T}$ method. Methods 25: 402-408, 2001.

Ma Y., Wang X.-P., Jiang H.-B., Shi D.-C.: Characteristics of organic acids accumulation and oxalate metabolism in Kochia sieversiana under salt and alkali stresses. - Acta Pratacult. Sin. 26: 158-165, 2017.

Munns R., Gilliham M.: Salinity tolerance of crops - what is the cost? - New Phytol. 208: 668-673, 2015.

Munns R., Tester M.: Mechanisms of salinity tolerance. - Annu. Rev. Plant Biol. 59: 651-681, 2008.

Rorat T.: Plant dehydrins - tissue location, structure and function. - Cell Mol. Biol. Lett. 11: 536-556, 2006.

Rozentsvet O.A., Nesterov V.N., Bogdanova E.S.: Structural, physiological, and biochemical aspects of salinity tolerance of halophytes. - Russ. J. Plant Physl+ 64: 464-477, 2017.

Shi W., Xu W., Li S. et al.: Responses of two rice cultivars differing in seedling-stage nitrogen use efficiency to growth under low-nitrogen conditions. - Plant Soil 326: 291-302, 2009.

Tanji K.K.E.: Agricultural Salinity Assessment and Management. Pp. 112. American Society of Civil Engineers, New York 1990.

Wang F., Chen Z.H., Liu X. et al.: The loss of RBOHD function modulates root adaptive responses to combined hypoxia and salinity stress in Arabidopsis. - Environ. Exp. Bot. 158: 125-135, 2019.

Wang F.W., Wang C., Sun Y. et al.: Overexpression of vacuolar proton pump ATPase ( $\mathrm{V}-\mathrm{H}^{+}$-ATPase) subunits $\mathrm{B}, \mathrm{C}$ and $\mathrm{H}$ confers tolerance to salt and saline-alkali stresses in transgenic alfalfa (Medicago sativa L.). - J. Integr. Agr. 15: 2279-2289, 2016.

Wang H., Ahan J., Wu Z. et al:: Alteration of nitrogen metabolism in rice variety 'Nipponbare' induced by alkali stress. - Plant Soil 355: 131-147, 2012.

Wang H., Lin X., Cao S., Wu Z.: Alkali tolerance in rice (Oryza sativa $\mathrm{L}$.): growth, photosynthesis, nitrogen metabolism, and ion homeostasis. - Photosynthetica 53: 55-65, 2015.

Wang L., Fang C., Wang K.: Physiological responses of Leymus chinensis to long-term salt, alkali and mixed salt-alkali stresses. - J. Plant Nutr. 38: 526-540, 2015.

Wang M., Xia G.: The landscape of molecular mechanisms for salt tolerance in wheat. - Crop J. 6: 42-47, 2018. 
Wang P., Cui Y.N., Gao L., Wang S.M.: Construction of RNAi expression vector of CYP86A gene in halophyte Puccinellia tenuiflora. - Acta Pratacult. Sin. 26: 105-110, 2017. [In Chinese]

Wang Y., Chu Y., Liu G. et al.: Identification of expressed sequence tags in an alkali grass (Puccinellia tenuiflora) cDNA library. - J. Plant Physiol. 164: 78-89, 2007.

Wang Y., Yang C., Liu G., Jiang J.: Development of a cDNA microarray to identify gene expression of Puccinellia tenuiflora under saline-alkali stress. - Plant Physiol. Bioch. 45: 567-576, 2007.

Wu H., Shabala L., Azzarello E.: $\mathrm{Na}^{+}$extrusion from the cytosol and tissue-specific $\mathrm{Na}^{+}$sequestration in roots confer differential salt stress tolerance between durum and bread wheat. - J. Exp. Bot. 69: 3987-4001, 2018.

Yang C., Shi D., Wang D.: Comparative effects of salt and alkali stresses on growth, osmotic adjustment and ionic balance of an alkali-resistant halophyte Suaeda glauca (Bge.). - Plant Growth Regul. 56: 179, 2008a.

Yang C., Chong J., Li C. et al.: Osmotic adjustment and ion balance traits of an alkali resistant halophyte Kochia sieversiana during adaptation to salt and alkali conditions. Plant Soil 294: 263-276, 2007.

Yang C., Wang P., Li C. et al.: Comparison of effects of salt and alkali stresses on the growth and photosynthesis of wheat. Photosynthetica 46: 107-114, 2008b.

Yin Z., Zhang H., Zhao Q. et al.: Physiological and comparative proteomic analyses of saline-alkali $\mathrm{NaHCO}_{3}$-responses in leaves of halophyte Puccinellia tenuiflora. - Plant Soil 437: 137-158, 2019.

Yu J.J., Chen S.X., Wang T. et al.: Comparative proteomic analysis of Puccinellia tenuiflora leaves under $\mathrm{Na}_{2} \mathrm{CO}_{3}$ stress. - Int. J. Mol. Sci. 14: 1740-1762, 2013.

Yu J.J., Chen S.X., Zhao Q. et al.: Physiological and proteomic analysis of salinity tolerance in Puccinellia tenuiflora. J. Proteome Res. 10: 3852-3870, 2011.

Zeng Y., Li Q., Wang H. et al.: Two NHX-type transporters from Helianthus tuberosus improve the tolerance of rice to salinity and nutrient deficiency stress. - Plant Biotechnol. J. 16: 310-321, 2018.

Zhai J., Dong Y., Sun Y. et al.: Discovery and analysis of microRNAs in Leymus chinensis under saline-alkali and drought stress using high-throughput sequencing. - PLoS ONE 9: e105417, 2014.

Zhang A., Zang W., Zhang X. et al.: Global proteomic mapping of alkali stress regulated molecular networks in Helianthus tuberosus L. - Plant Soil 409: 175-202, 2016.

Zhang W.-D., Wang P., Bao Z. et al.: SOS1, HKT1;5, and NHX1 synergistically modulate $\mathrm{Na}^{+}$homeostasis in the halophytic grass Puccinellia tenuiflora. - Front. Plant Sci. 8: 576, 2017.

Zhang M., Cao Y., Wang Z. et al.: A retrotransposon in an HKT1 family sodium transporter causes variation of leaf $\mathrm{Na}^{+}$ exclusion and salt tolerance in maize. - New Phytol. 217: 1161-1176, 2018.

Zhang X., Wei L., Wang Z., Wang T.: Physiological and molecular features of Puccinellia tenuiflora tolerating salt and alkalinesalt stress. - J. Integr. Plant Biol. 55: 262-276, 2013.

Zhao L., Yang Z., Yang C.: Transcriptomic profiling and physiological responses of halophyte Kochia sieversiana provide insights into salt tolerance. - Front. Plant Sci. 8: 1985, 2017.

Zhao Z., Liu J., Jia R. et al.: Physiological and TMT-based proteomic analysis of oat early seedlings in response to alkali stress. - J. Proteomics 193: 10-26, 2019.

Zheng H.Y., Li J.D.: [Form and dynamic trait of halophyte community.] - In: Zheng H.Y., Li J.D. (ed.): [Saline Plants in Songnen Plain and Restoration of Alkaline-Saline Grass.] Pp. 137-138. Science Press, Beijing 1999. [In Chinese]

Zhu D., Hou L., Xiao P. et al.: VvWRKY30, a grape WRKY transcription factor, plays a positive regulatory role under salinity stress. - Plant Sci. 280: 132-142, 2019.

Zhu G.L.: Carotenoid and chlorophyll determination. - In: Zhu G.L. (ed.): [Laboratory Manual of Plant Physiology.] Pp. 51-54. Beijing University Press, Beijing 1993. [In Chinese]

(C) The authors. This is an open access article distributed under the terms of the Creative Commons BY-NC-ND Licence. 